\title{
Development of an in vitro Biochemical Assay System for the Measurement of Residual Toxin Activities in Pertussis Toxin Containing Vaccines
}

Chun-Ting Yuen ${ }^{1}$, Catpagavalli Asokanathan ${ }^{2 *}$, Alexandra Douglas-Bardsley ${ }^{2}$, Kevin Markey $^{2}$, Peter Rigsby ${ }^{1}$ and Dorothy Xing ${ }^{2}$

${ }^{1} T D I$, Blanche Lane, South Mimms, Potters Bar, Hertfordshire, UK

2 Division of Bacteriology, National Institute for Biological Standards and Control (NIBSC), Blanche Lane, South Mimms, Potters Bar, EN6 3QG, UK

"Corresponding Author: Catpagavalli Asokanathan, Division of Bacteriology, Blanche Lane, South Mimms, Potters Bar, Hertfordshire, UK, Tel: 4401707 641000; Fax: 4401707641054; E-mail: Cathy.Asokanathan@nibsc.org

Received date: February 15, 2017; Accepted date: March 22, 2017; Published date: March 28, 2017

Copyright: (C) 2017 Yuen CT, et al. This is an open-access article distributed under the terms of the Creative Commons Attribution License, which permits unrestricted use, distribution, and reproduction in any medium, provided the original author and source are credited.

\begin{abstract}
Pertussis (whooping cough) vaccines play an essential role in immunisation programmes worldwide and the histamine sensitisation test (HIST) is currently the official batch-release test for acellular pertussis containing combination vaccines. HIST, being a murine animal test, has large assay variability problems and ethical issues. Therefore, in response to the $3 R$ s, a biochemical assay system has been developed based on the pertussis toxin (PTx)'s two functional domains: an enzyme active A-protomer and a carbohydrate binding B-oligomer. The clinical relevance of these two biochemical tests in relation to the PTx toxic effects has been demonstrated. In order to assess the practicality of this assay system, an international collaborative study involving 9 countries on method transferability had also been performed and showed that this assay system could be adopted readily in control laboratories and vaccine manufacturing facilities. In this update, we provide further optimised assay conditions for measuring both the residual PTx ADP-ribosyltransferase and carbohydrate binding activities in specific vaccines. In general, appropriate dilution of vaccines would help to increase test sensitivity. Spiking experiments showed carbohydrate binding assay could detect low levels of PTx similar to HIST. Although positive trend with increasing spikes could also be demonstrated in ADP-ribosyltransferase activity assay for some vaccines, the resultant values in a number of spiked vaccines were not statistically significant and this is due to high baseline activity of the unspiked vaccines. Since all the reagents of this biochemical assay system are available worldwide, readily controlled and with good test consistency, both assays could be used for monitoring product consistency. The carbohydrate binding assay could even be considered as a potential alternative to HIST for batch-release of acellular pertussis vaccines. Protocols for this in vitro assay system are supplied as Supplement Documents $1 \& 2$.
\end{abstract}

Keywords: Acellular pertussis vaccine; Histamine sensitisation test (HIST); In vitro alternative; Carbohydrate-binding; Enzyme-HPLC; Pertussis toxin

Abbreviations AsF: Asialofetuin; BSA: Bovine Serum Albumin; BU: Carbohydrate Binding Unit; D: Diphtheria Toxoid; EDTA: Ethylene Diamine Tetra-Acetic Acid; EU: ADP-Ribosyltransferase Enzymatic Unit; Fet: Fetuin; FHA: Filamentous Haemagglutinin; Fims2/3: Fimbriae Type 2 and Type 3; F: Formaldehyde; g-PTd: Genetically Inactivated PTx Toxoid; G: Glutaraldehyde; Hib: Haemophilus Influenzae Type b; HPLC: High Performance Liquid Chromatography; HIST: Histamine Sensitisation Test; HRP: Horseradish Peroxidase; $\mathrm{H}_{2} \mathrm{O}_{2}$ : Hydrogen Peroxide; IPV: Inactivated Poliovirus Vaccine; i.p.: Intra-Peritoneal; mAb: Monoclonal Antibody; OD: Optical Density; PRN: Pertactin; PTx: Pertussis Toxin; PTd: Pertussis Toxoid; PBS Phosphate Buffered Saline; PBSG: PBS Gelatine; pAb: Polyclonal Antibody; rcf: Relative Centrifugation Force; SHD: Single Human Dose; T: Tetanus Toxoid; WHO: World Health Organisation.

\section{Introduction}

Whooping cough caused by Bordetella pertussis infection is a serious disease and could result in morbidity and fatality especially for infants and young children. Pertussis toxin (PTx) has a wide range of physio-pathological effects including induction of leucocytosis, histamine-sensitisation, increased insulin production with consequent hypoglycaemia, potentiation of anaphylaxis and lethality in mice and it has also been reported to play a possible role in neuropathology $[1,2]$. Two types of pertussis vaccines are recommended by WHO for immunisation campaigns worldwide: whole-cell (WCV) and acellular pertussis vaccines (ACV) [3]. Chemically detoxified pertussis toxin (PTd) is the common and major protective antigen present in these vaccines and the monitoring of their safety due to residual toxicity and/or reversion to toxicity is an essential task for all regulatory authorities worldwide [4-7]. In vivo histamine sensitisation test (HIST) is currently the official batch-release test for ACVs and there are two versions: one is based on lethal end point, which is widely used in North American and European countries [6,7] and the other alternative measures temperature reduction after histamine challenge $[8,9]$ and this is used successfully in Japan and other countries [10]. The lethal end point method and its disadvantages have already been reviewed [11]. Although the temperature method provides a more precise quantitative estimate for the HIST and hence uses less animals, the precise mechanism of HIST is unclear and it is still difficult to standardise [12]. Therefore, there is an urgent need to find an in vitro alternative for HIST in response to the 3Rs (Replacement, Reduction and Refinement of testing with animals).

PTx molecule has an A-B type structure typical of many other bacterial toxins by having an enzyme active A-protomer (monomer), the S-1 subunit and a carbohydrate binding B-oligomer of subunits S-2 through to S-5 [13-15]. Intact B-oligomer is required for binding of the holotoxin to receptor sites on the cell surface and enables entry of the 
Citation: Yuen CT, Asokanathan C, Douglas-Bardsley A, Markey K, Rigsby P, et al. (2017) Development of an in vitro Biochemical Assay System for the Measurement of Residual Toxin Activities in Pertussis Toxin Containing Vaccines. J Vaccines Vaccin 8: 355. doi: $10.4172 / 2157-7560.1000355$

Page 2 of 6

A-protomer into the cells [16]. Cyr et al. of Health Canada first developed an enzyme-coupled HPLC (E-HPLC) assay procedure to measure the A-protomer ADP-ribosyltransferase activities in vaccine products with the aim to replace HIST [17]. NIBSC scientists, in collaboration with Health Canada, modified the E-HPLC further for easier method transfer between laboratories [18]. The E-HPLC method was later included in one of the European Directorate for the Quality of Medicine (EDQM) Biological Standardisation Programmes, BSP032 (2001) in an international collaborative study for the establishment of a European Pharmacopoeia (EP) Biological Reference Preparation (BRP) for Pertussis Toxin [19]. However, during the course of this programme and further studies, it was recognised that ADPribosyltransferase activity alone could not correlate with residual PTx toxicity found by HIST in vaccines [20]. It has also been demonstrated that this enzyme activity is vaccine product specific and there is a wide range of baseline activities between vaccine products. In order to overcome the limitation of the E-HPLC, a complementary assay, CarbELISA, which measures the cell binding activity of the B-oligomer to a glycoprotein coat was developed [21]. This biochemical assay system was successfully validated and results correlated positively with HIST in the measurement of residual PTx activities in different types of vaccines [22]. Subsequently, on behalf of WHO, an international collaborative study on method transfer was conducted to analyse 3 common commercially available but different types of ACVs, involving 16 labs in 9 countries [23]. Results showed that the biochemical assay system was transferable between laboratories worldwide and was suitable for the three types of ACV products used in the study. Same activity rank orders among the three types of vaccines were also demonstrated between the in vitro assay system and the in vivo HIST.

Although this in vitro assay system was cited as a "small prints" note as a potential alternative to the current HIST in "WHO Recommendations to Assure the Quality, Safety and Efficacy of Acellular Pertussis Vaccines" (2011) [24], the uptake and adoption of this assay system is limited as there are concerns with this cell free assay approach. The main criticism is twofold: (1) The mechanism of PTx actions involves host cell binding, translocation of the PTx into the cell and then subsequent adverse cell functions due to enzymatic reaction. The in vitro biochemical assay system only measures binding and enzymatic activities but does not measure the translocation stage; (2) The assay sensitivity has not been demonstrated, such as via using PTx spikes. To answer the first point, a set of cell based assays was conducted using confocal microscopic method and indirect immunofluorescence detection to investigate the interaction of PTx and different types of PTd with CHO cells [25]. Translocation/ internalization of PTx and PTd were observed and the relative intensity of entry correlated positively with the Carb-ELISA binding activities of the test products. This indicates that the carbohydrate binding activity reflects the degree of translocation/internalization activity of PTx and PTd. Furthermore, in a separate in vivo study using mouse footpad swelling model, Carb-ELISA binding activity in ACVs showed a positive relationship with the severity of local reactions after booster vaccination [26]. This again emphasises the role of the B-oligomer and as an important determinant for PTx toxicity. In order to address the assay sensitivity and to simulate the conditions of a failed batch (there were no "failed vaccines" available for this study), we set up PTx spiking experiments including the consensus minimum spike level of 2 IU which had been discussed and agreed in meetings at the International HIST Alternative Working Group [27,28]. Here we report our results and discuss further the possible application of this in vitro method in the safety monitoring of $\mathrm{PTx} / \mathrm{PTd}$ containing vaccines.

\section{Materials and Methods}

\section{Materials}

The PTx (code 90/518) used was a freeze-dried reference preparation from the National Institute for Biological Standards and Control, UK (NIBSC), stored at $-20^{\circ} \mathrm{C}$. Each ampoule contained a nominal $20 \mu \mathrm{g}$ of PTx, which was equivalent to $2100 \mathrm{IU}$ of PTx activity measured by HIST (lethal endpoint method) when calibrated against the WHO First International Standard for Pertussis Toxin (NIBSC Code JNIH-5) [29]. For use, each ampoule of 90/518 was reconstituted with $10 \mathrm{ml}$ ovalbumin solution ( $2 \mathrm{mg} / \mathrm{ml}$; Sigma, A5503) in PBS to give a $2 \mu \mathrm{g} \mathrm{PTx} / \mathrm{ml}$ solution and aliquots of $100 \mu \mathrm{l}(200 \mathrm{ng})$ were lyophilised and stored at $-20^{\circ} \mathrm{C}$ as stock standards. A two-component ACV, containing PTd and filamentous haemagglutinin (FHA) in combination with diphtheria (D) and tetanus (T) toxoids (NIBSC code 00/486) was kindly donated by the Research Foundation for Microbial Diseases of Osaka University (Biken, Japan) and freeze-dried at NIBSC (nominal potency $11.25 \mathrm{IU} /$ vial). This product was used as a positive control in the E-HPLC assay.

Test vaccines were commercially available or kindly donated for collaborative studies by Sanofi Pasteur (Canada), GlaxoSmithKline Biologicals (Belgium) and Takeda Pharmaceutical (Japan). They comprised PTd in combination with $\mathrm{D}$ and T toxoids (DTaP) \pm other antigens such as Haemophilus influenzae type $\mathrm{b}$ capsular polysaccharide conjugated to $\mathrm{T}$ (Hib), inactivated polio vaccine (IPV) and other B. pertussis antigens (filamentous haemagglutinin (FHA), pertactin (69K) and fimbriae 2 and 3 (Fims 2/3)). The adjuvant in vaccines is either aluminium phosphate or aluminium hydroxide.

The fluorescein-tagged PTx peptide substrate, FVFDAVTDVIIKNNLKECGLY-COOH (F-G ${ }_{\alpha i 3} \mathrm{C} 20$, HCAM-2) was custom-synthesised by AnaSpec Inc., San Jose, CA, USA and was reported to have $>95 \%$ purity. Anti-PT sheep serum (Code: 97/572) was prepared at NIBSC. Horseradish peroxidase labelled anti-sheep IgG (A-3415) was obtained from Sigma. All other chemicals, unless specified otherwise, were of analytical grade and purchased from either Sigma-Aldrich or VWR-BDH (Poole, UK).

\section{E-HPLC ADP-ribosyltransferase assay and Carb-ELISA carbohydrate binding assay}

The determination of PTx ADP-ribosyltransferase activity in vaccines was performed using a synthetic fluorescein-tagged peptide substrate, $\mathrm{F}-\mathrm{G}_{\mathrm{ai} 3} \mathrm{C} 20$ coupled with an HPLC method for the separation and quantitation of the ADP-ribosylated product. The PTx carbohydrate-binding activity in vaccines was carried out using bovine fetuin (Sigma, F3004) as carbohydrate ligand. Both assays had been described in detail previously [18,21-23] and protocols for the two assays are supplied here as Supplement Documents 1 and 2. The enzyme and carbohydrate binding activities were expressed as EU and $\mathrm{BU}$ respectively where $1 \mathrm{EU}$ or $1 \mathrm{BU}$ was equivalent to the corresponding activity observed by $1 \mu \mathrm{g}$ PTx under the same assay conditions.

\section{Dermal temperature histamine sensitisation test (HIST)}

The HIST was carried out according to the method described by Ochiai et al. [8]. In brief, NIH female mice weighing 9-11 grams were randomly distributed in groups of 10 (caged in 5's and individually marked). Mice were inoculated intra-peritoneally (i.p.) with either 0.5 
Citation: Yuen CT, Asokanathan C, Douglas-Bardsley A, Markey K, Rigsby P, et al. (2017) Development of an in vitro Biochemical Assay System for the Measurement of Residual Toxin Activities in Pertussis Toxin Containing Vaccines. J Vaccines Vaccin 8: 355. doi: $10.4172 / 2157-7560.1000355$

Page 3 of 6

$\mathrm{ml}$ PBS containing $0.2 \%(\mathrm{w} / \mathrm{v})$ gelatine (PBSG) as negative control, PTx reference (BRP) in PBSG at doses 10, 5 and 1 IU/dose or with test vaccine at 1 single human dose (SHD, $0.5 \mathrm{ml}) /$ mouse. Five days after inoculation, mice were challenged i.p. with $0.5 \mathrm{ml}$ of histamine solution (equivalent to $2 \mathrm{mg}$ histamine base per dose prepared in PBS). The dermal temperature of each mouse was measured 30 minutes after challenge using an infrared thermometer (IT-550, Horiba Ltd, Japan). The HIST activity in the test vaccine was calculated in comparison with that of the reference toxin groups and expressed as IU/dose.

\section{Ethical Approval}

Ethical approval was obtained for all animal experiments from the Ethical Committee of National Institute for Biological Standards and Control, UK. All experiments were regulated according to the Animal Scientific Procedures Act (1986), UK.

\section{Statistical Analysis}

The relative potency of PTd binding activity in vaccine samples to the PTx reference under identical assay conditions was calculated by parallel line assay analysis via the CombiStats software (version 4.0, EDQM). Data distribution analysis was by Minitab 17 software and Student's T-test was used to compare the difference of bioactivities between groups. Statistical significance was concluded at the $5 \%$ level $(\mathrm{p}<0.05)$.

\section{Results}

As shown in previous studies, a wide range of enzyme activities and carbohydrate binding activities were found in specific vaccines with $\mathrm{E}$ HPLC values ranging from 0.08 to $1.30 \mathrm{EU} / \mathrm{ml}$ (Figure 1A) and CarbELISA with values ranging from $0.01-0.50 \mathrm{BU} / \mathrm{ml}$ (Figure 1B). It is important to note that both activities might not be related proportionally as different chemical detoxification of PTx would affect differently to the PTx subunits and it also depends on other factors such as detoxification reagent types, reaction conditions and formulation processes [30,31].

\section{PTx Spiking Experiments}

\section{Carb-ELISA}

In the original assay procedure, neat vaccines after desorption were used in the spiking assays. Mean positive dose responses for $2 \mathrm{IU}, 10$ $\mathrm{IU}$ and $20 \mathrm{IU}$ were observed for five out of six commercial vaccines of four different formulations (Figure 2A). The regressions (slopes of log transformed response against log spike IU) are significant for $\mathrm{vA}, \mathrm{vB}$, $\mathrm{vE}$ and $\mathrm{vF}(\mathrm{p}<0.001)$ and $\mathrm{vD}(\mathrm{p}=0.010)$ but not $\mathrm{vC}(\mathrm{p}=0.362)$. The detection of the 2 IU spike was greatest in vaccines with low baseline binding activities such as in $\mathrm{vA}, \mathrm{vB}$ and $\mathrm{vF}$ (Figure $1 \mathrm{~B}$ ). For $\mathrm{vC}$, the differences between spikes were not significant because of its high baseline binding activity which masked the small increases in spikes. The introduction of a pre-dilution step in the assay of $\mathrm{vC}$ samples (e.g. 1:3 dilutions) with PBS prior to the binding assay had improved the detection sensitivity as well as the differentiation between spikes comparing to the results when neat vaccines were tested. An example of $\mathrm{vC}$ spiked and un-spiked with PTx is shown in Figure 2B; the detection of $2 \mathrm{IU}$ to un-spiked is significant: $\mathrm{p}<0.001$.



Figure 1: Baseline PTx enzyme and carbohydrate binding activities in different ACVs. Different acellular pertussis containing vaccine products have different specific baseline ADP-ribosyltransferase (Panel A) and carbohydrate binding activities (Panel B). The baseline values of individual vaccines were obtained from assays of one or more batches of each type of vaccines. They were performed in duplicates for E-HPLC (Panel A) and triplicates for Carb-ELISA (Panel B).

Two vaccines (a batch each from type vA and vC) were compared with their behaviour in HIST and in Carb-ELISA tests after PTx spiking (Table 1). Both vaccines showed increases in activities with increasing spikes in both assay systems (Columns BU/dose and IU/ dose (HIST)). Distinctive baseline binding activities were also found by both methods between the vaccines (First row, Table 1). It is worth noting that both the in vitro and in vivo assay system showed that vA was less responsive to spikes compared to $\mathrm{vC}$. This is probably due to different adjuvants used in the vaccines where vA contains aluminium hydroxide while vC contains aluminium phosphate. Since the toxin activity unitage for HIST and Carb-ELISA are defined under different criteria and assay conditions, direct correlation between the two methods would be inappropriate. Nonetheless, similar positive dose response trend was observed in these two assays. 
Citation: Yuen CT, Asokanathan C, Douglas-Bardsley A, Markey K, Rigsby P, et al. (2017) Development of an in vitro Biochemical Assay System for the Measurement of Residual Toxin Activities in Pertussis Toxin Containing Vaccines. J Vaccines Vaccin 8: 355. doi: $10.4172 / 2157-7560.1000355$

Page 4 of 6

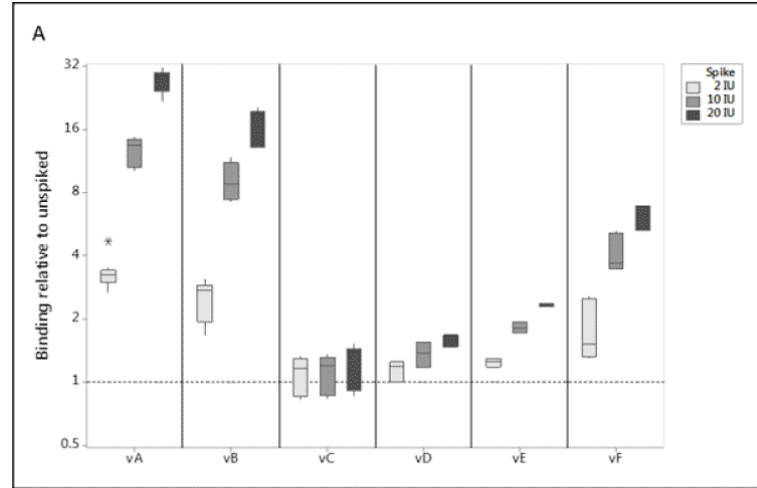

B $\quad v C(1: 3$ dilution prior to assay; $n=4 \times 3)$

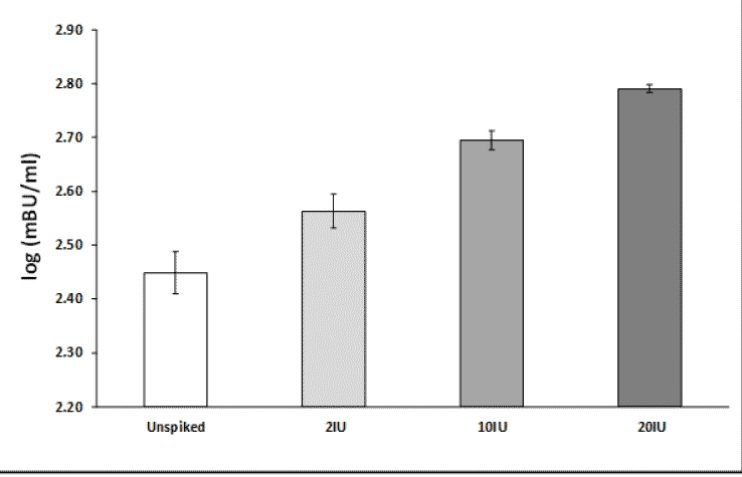

Figure 2: Panel A. Data distribution analysis of Carb-ELISA of vaccines spiked with reference PTx (2, 10 and $20 \mathrm{IU})$. Six vaccines with different formulations were spiked with reference PTx and assayed using neat vaccines after desorption. The numbers of assays in triplicates for each vaccine are as follow: vA $(3 \times 3), \mathrm{vB}(3 \times 3)$, vC $(3 \times 3), v D(1 \times 3), v E(1 \times 3)$ and $\mathrm{vF}(2 \times 3)$. Spike response was plotted relative to the un-spiked value. The regressions (slopes) are significant for $\mathrm{vA}, \mathrm{vB}, \mathrm{vE}$ and $\mathrm{vF}(\mathrm{p}<0.001)$ and $\mathrm{vD}(\mathrm{p}=0.01)$ but not $\mathrm{vC}(\mathrm{p}=0.362)$.

\begin{tabular}{|c|c|c|c|c|c|c|c|}
\hline Vaccine & $\begin{array}{l}\text { Spike } \\
\text { (IU) }\end{array}$ & $\begin{array}{l}\text { BU/ } \\
\text { dose }\end{array}$ & $\begin{array}{l}\text { IU/dose } \\
\text { (HIST) }\end{array}$ & Vaccine & $\begin{array}{l}\text { Spik } \\
\text { e (IU) }\end{array}$ & $\begin{array}{l}\text { BU/ } \\
\text { dose }\end{array}$ & $\begin{array}{l}\text { IU/dose } \\
\text { (HIST) }\end{array}$ \\
\hline \multirow[t]{6}{*}{ vaccine $A$} & 0 & 0.005 & 0.02 & \multirow[t]{6}{*}{ vaccine $\mathrm{C}$} & 0 & 0.1 & 0.2 \\
\hline & 1 & - & 1.61 & & 1 & - & 3.87 \\
\hline & 2 & 0.021 & - & & 2 & 0.122 & - \\
\hline & 5 & - & 2.15 & & 5 & - & 5.86 \\
\hline & 10 & 0.053 & 2.96 & & 10 & 0.175 & 8.55 \\
\hline & 20 & 0.071 & - & & 20 & 0.209 & - \\
\hline
\end{tabular}

Table 1: Behaviour of vA, which contains aluminium hydroxide and $\mathrm{vC}$, which contains aluminium phosphate after PTx spiking when analysed by Carb-ELISA and HIST (Temp). The data are represented as binding activity (BU/dose) and HIST (IU/dose) where 1 dose equals $0.5 \mathrm{ml}$ of vaccine.

\section{E-HPLC}

In this method, all vaccines were pre-diluted 5 to 20 times with OVS before assay to keep the activity values within range of the PTx working standard curve $(0-400 \mathrm{ng} \mathrm{PTx} / \mathrm{ml})$. Only vaccines with baseline enzyme activities lower than $0.1 \mathrm{EU} / \mathrm{ml}$ showed good positive relationship with increasing spikes, for example, Vaccine vA: p-values for $2 \mathrm{IU}, 10 \mathrm{IU}$ and $20 \mathrm{IU}$ spikes to un-spiked are 0.510, 0.004 and $<0.001$ respectively. As for the high baseline vaccines, for example vB at levels $\sim 0.3 \mathrm{EU} / \mathrm{ml}$ and of those even higher (data not shown), the spikes differences were not distinguishable (Figure 3).

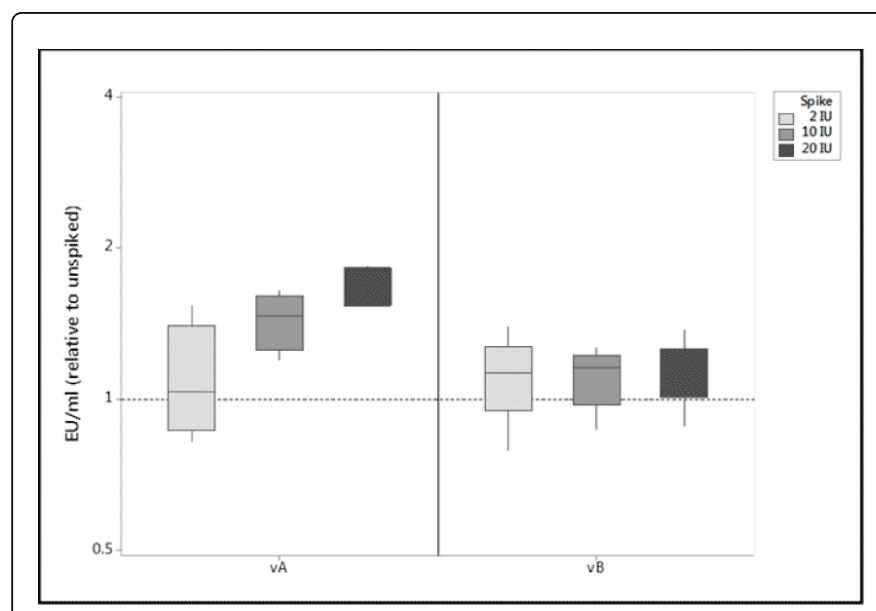

Figure 3: Data distribution analysis of ADP-ribosyltransferase activities in PTx spiked vaccines. $\mathrm{vA}$ and $\mathrm{vB}$ are vaccines from different manufacturers with different formulation and each vaccine was analysed twice in duplicates. Spike responses were plotted relative to the un-spiked value.

\section{Discussion}

PTx is an $\mathrm{AB}$ type bacterial toxin and has two distinctive functional domains: the enzymatic subunit S1 (A-protomer), which is an ADPribosyltransferase and the subunits S2-5 (B-oligomer), which is a carbohydrate binding molecule that facilitates host cell binding and entry of PTx into the cell [30]. Ideally, PTx in pertussis vaccines should be completely detoxified while still preserving immunogenicity. However, this may not always be the case and an in vivo HIST for testing its safety level is required by regulatory authorities before they can be released to the public. In view of the disadvantages of the current in vivo HIST and in response to the $3 \mathrm{Rs}$, an in vitro biochemical assay system targeting the PTx enzymatic and carbohydrate binding activities has been developed as a potential alternative [22]. In a previous study, the two biochemical activities have been shown to be valuable to assess functional changes in residual PTx activities after different chemical detoxification and the resultant biochemical activities are vaccine manufacturing and type specific [31].

Although this in vitro biochemical assay system has been shown to have positive relationship to the in vivo HIST [22] and the assay methods could be transferred readily worldwide [23], there is still a reluctance to adopt this method as an alternative due to its intrinsic assay principle as compared to cell based assay systems. Such concern for this biochemical assay system is real and to answer the critics, two 
Citation: Yuen CT, Asokanathan C, Douglas-Bardsley A, Markey K, Rigsby P, et al. (2017) Development of an in vitro Biochemical Assay System for the Measurement of Residual Toxin Activities in Pertussis Toxin Containing Vaccines. J Vaccines Vaccin 8: 355. doi: $10.4172 / 2157-7560.1000355$

Page 5 of 6

studies were successfully performed to demonstrate: (1) positive relationship of the Carb-ELISA activities with PTx/PTd translocation and internalisation into target $\mathrm{CHO}$ cells [25] and (2) positive relationship of Carb-ELISA binding activities with severity of in vivo local reaction after pertussis ACV booster injection [26]. Both studies confirmed PTx carbohydrate binding activities could be used as an indicator of PTx toxicity. This is not surprising as PTx binding is considered to be the first step in the mechanism of toxin effect. The other uncertainty of the biochemical assay system was about its sensitivity to detect faulty vaccines. A level of 2 IU PTx activity detected by HIST was in the range of residual PTx activities found in current commercial vaccines in an international collaborative study in 2010 and these vaccines were considered safe [27]. The sensitivity of an assay method could be demonstrated by spiking experiments. Hence in this report, we provide data that the Carb-ELISA could detect 2 IU spikes in five out of six common commercial vaccines using a standard protocol and in the case of a vaccine product $(\mathrm{vC})$ which has high baseline activity, the 2 IU spikes can be detected by using the modified protocol. Although ADP-ribosyltransferase activity could show positive trend with spikes, detection was not significant in many test vaccines because of their relatively high baseline activity. Nonetheless, with appropriate assay criteria and assay standards, both methods could be used to monitor product consistency during and after production. Since the Carb-ELISA activities also shows positive relationship to PTx toxicity such as in the mouse footpad local reaction [26] and toxin/toxoid translocation into target cells [25], this test could even be considered as a clinically relevant functional safety test similar to HIST for batch-release of ACVs. The advantage of the biochemical assays is that all the assay components can be readily controlled or standardised. For interested parties, protocols of both Carb-ELISA and E-HPLC are provided as Supplement Documents for reference and for use.

\section{Conclusion}

We have demonstrated that the developed biochemical PTx assay system that consists of the measurement of ADP-ribosyltransferase and carbohydrate binding activities is capable of estimating the activities of residual PTx functional domains that accounts for its toxicity in chemically detoxified PT vaccines. In addition, all the assay components of this test can be readily controlled and standardised with minimal problems of reagents availability worldwide or quality issues such as in most cell based assays. Therefore, we would recommend interested parties such as ACV vaccine manufacturers and regulators to try out this test system alongside the current HIST to validate and possibly to establish the relationship between the in vitro and in vivo assays. Protocols of both Carb-ELISA and E-HPLC are provided as Supplement Documents for reference and for use.

\section{Acknowledgements}

We are grateful for the support provided by the Biological Services Division at NIBSC. We also acknowledge APC, Home Office and the National Centre for 3Rs, UK for providing funds to support the research and development of the in vitro assay system. Great thanks to WHO and all participants for supporting and for their invaluable contribution to the international collaborative study of method transfer of the biochemical assay. Special acknowledgements are due to Sanofi Pasteur Ltd, Canada, GlaxoSmithKline Biologicals, Belgium and Takeda Pharmaceutical Company Ltd., Japan for their supports and generous provision of vaccine samples. Lastly, we would also give special thanks to all our collaborators in this long running project.

\section{Authors' Contribution}

CT. Yuen and D. Xing contributed to the concept, design, analyses, interpretation of the study and drafting of this manuscript. C. Asokanathan and A. Douglas-Bardsley developed, optimised, performed and analysed all the Carb-ELISA assays. CT. Yuen and K. Markey developed, optimised, performed and analysed all the E-HPLC assays. C. Asokanathan and D. Xing performed all the in vivo HIST. P. Rigsby performed and advised on all statistical analyses in collaborative studies and studies at NIBSC.

\section{References}

1. Munoz JJ, Sekura RD, Moss J, Vaughan M (1985) Biological activities of pertussigen (pertussis toxin). Pertussis toxin: London, Academic Press Inc., pp: 1-18.

2. Miller D, Madge N, Diamond J, Wadsworth J, Ross E (1993) Pertussis immunisation and serious acute neurological illnesses in children. BMJ 307: 1171-1176.

3. WHO (2005) Pertussis vaccines. WHO position paper: Weekly Epidemiological Record 80: 29-40.

4. Kaketsuken (2004) Adsorbed diphtheria-purified pertussis-tetanus combined vaccine, In: Minimum requirements for Biological products; Ministry of Health, Japan, Labour and Welfare pp: 41-49.

5. Arciniega JL, Corbel M, Dellepiane N, Dobbelaer R, Griffiths E (1998) WHO guidelines for the production and control of the acellular pertussis component of monovalent or combined vaccines. In: WHO Technical Report Series 878: 57-72.

6. Title 21, Pertussis vaccine 620.1. In: U.S. Code of Federal Regulations. Washington DC, U.S. Government Printing Office. 1983: 58-61.

7. EP (2008) Pertussis vaccine (acellular, component, adsorbed), In: European Pharmacopoeia, version 7.0, 1356: 806-807.

8. Ochiai M, Yamamoto A, Kataoka M, Toyoizumi H, Arakawa Y, et al. (2007) Highly sensitive histamine-sensitization test for residual activity of pertussis toxin in acellular pertussis vaccine. Biologicals 35: 259-64.

9. Gaines-Das R, Ochiai M, Douglas-Bardsley A, Asokanathan C, Horiuchi $\mathrm{Y}$, et al. (2009) Transferability of dermal temperature histamine sensitisation test for estimation of pertussis toxin activity in vaccines. Human Vaccines 5: 166-171.

10. Chinese Pharmacopoeia (2005) Pertussis vaccine, Beijing, People's Medical Publishing House 3: 67-71.

11. Corbel MJ, Xing DK (2004) Toxicity and potency evaluation of pertussis vaccines. Expert Rev Vaccines 3: 89-101.

12. van Straaten-van de Kappelle I, van der Gun JW, Marsman FR, Hendriksen CF, van de Donk HJ (1997) Collaborative study on test systems to assess toxicity of whole cell pertussis vaccine. Biologicals 25 : 41-57.

13. Tamura M, Nogimori K, Murai S, Yajima M, IK, Katada T et al. (1992) Subunit structure of islet-activating protein, pertussis toxin, in conformity with the A-B model, Biochemistry 21: 5516-5522.

14. Tamura M, Nogimori K, Yajima M, Ase K, Ui M (1983) A role of the Boligomer moiety of islet-activating protein, pertussis toxin, in development of the biological effects on intact cells. The Journal of Biological Chemistry 258: 6756-6761.

15. Sekura RD, Fish F, Manclark CR, Meade B, Zhang YL (1983) Pertussis toxin. Affinity purification of a new ADP-ribosyltransferase. J Biol Chem 258: 14647-14651.

16. Bourne HR (1990) ADP-Ribosylating Toxins and G Proteins. Insights into Signal Transduction. Joel Moss and Martha Vaughan, Eds. American Society for Microbiology, Washington, DC, 1990. xviii, 567 pp., illus. \$79; to ASM members, \$69. Science 250: 841-842. 
Citation: Yuen CT, Asokanathan C, Douglas-Bardsley A, Markey K, Rigsby P, et al. (2017) Development of an in vitro Biochemical Assay System for the Measurement of Residual Toxin Activities in Pertussis Toxin Containing Vaccines. J Vaccines Vaccin 8: 355. doi: $10.4172 / 2157-7560.1000355$

Page 6 of 6

17. Cyr T, Menzies AJ, Calver J, Whitehouse LW (2001) A quantitative analysis for the ADP-ribosylation activity of pertussis toxin: an enzymatic-HPLC coupled assay applicable to formulated whole cell and acellular pertussis vaccine products. Biologicals 29: 81-95.

18. Yuen CT, Canthaboo C, Menzies JA, Cyr T, Whitehouse LW (2002) Determination of residual pertussis toxin in vaccines using a modified ribosylation assay. Vaccine 21: 44-52.

19. Xing D, Maes A, Behr-Gross ME, Buchheit KH (2010) Collaborative Study for the Establishment of a European Pharmacopoeia Biological Reference Preparation for Pertussis Toxin.

20. Gomez SR, Yuen CT, Asokanathan C, Douglas-Bardsley A, Corbel MJ, et al. (2007) ADP-ribosylation activity in pertussis vaccines and its relationship to the in vivo histamine-sensitisation test. Vaccine 25: 3311-3318.

21. Gomez SR, Xing DK, Corbel MJ, Coote J, Parton R, et al. (2006) Development of a carbohydrate binding assay for the B-oligomer of pertussis toxin and toxoid. Anal Biochem 356: 244-253.

22. Yuen CT, Canthaboo C, Menzies JA, Cyr T, Whitehouse LW, et al. (2010) An in vitro assay system as a potential replacement for the histamine sensitisation test for acellular pertussis based combination vaccines. Vaccine 28: 3714-3721.

23. Xing D, Yuen CT, Asokanathan C, Rigsby P, Horiuchi Y (2012) Evaluation of an in vitro assay system as a potential alternative to current histamine sensitization test for acellular pertussis vaccines. Biologicals 40: 456-465.

24. WHO (2011) Recommendations to Assure the Quality, Safety and Efficacy of Acellular Pertussis Vaccines. WHO BS2158.

25. Tan Y, Fleck RA, Asokanathan C, Yuen CT, Xing D (2013) Confocal microscopy study of pertussis toxin and toxoids on CHO-cells. Vaccines \& Immunotherapeutics 9: 332-338.
26. Ochiai M, Horiuchi Y, Yuen CT, Asokanathan C, Yamamoto A, et al. (2014) Investigation in a murine model of possible mechanisms of enhanced local reactions to post-primary diphtheria-tetanus toxoid boosters in recipients of acellular pertussis, diphtheria-tetanus vaccine. Human Vaccines \& Immunotherapeutics 10: 2074-2080.

27. Xing D, Maes A, Behr-Gross ME, Costanzo A, Daas A, et al. (2010) Collaborative study for the standardisation of the histamine sensitizing test in mice and the $\mathrm{CHO}$ cell-based assay for the residual toxicity testing of acellular pertussis vaccines. Pharmeur Bio Sci Notes pp: 51-63.

28. Arciniega J, Wagner L, Prymula R, Sebo P, Isbrucker R, et al. (2016) Alternatives to HIST for acellular pertussis vaccines: progress and challenges in replacement. Report on an international workshop. Pharmeur Bio Sci Notes pp: 82-96.

29. Xing D, Gaines-Das R, Newland P, Corbel M (2002) Comparison of the bioactivity of reference preparations for assaying Bordetella pertussis toxin activity in vaccines by the histamine sensitisation and Chinese hamster ovary-cell tests: assessment of validity of expression of activity in terms of protein concentration. Vaccine 20: 3535-3542.

30. Locht C, Antoine R, Alouf JE, Freer JH (1999) Bordetella pertussis protein toxins. In: Bacterial protein toxins. (2nd Edn) London, Academic Press Inc. pp: 130-146.

31. Yuen CT, Asokanathan C, Cook S, Lin N, Xing D (2016) Effect of different detoxification procedures on the residual pertussis toxin activities in vaccines Vaccine 34: 2129-2134. 